\title{
Proposta para identificação rápida dos períodos hidrológicos em áreas de várzea do rio Solimóes-Amazonas nas proximidades de Manaus
}

\author{
Maria Mercedes BITTENCOURT ${ }^{1}$, Sidinéia Aparecida AMADIO²
}

\section{RESUMO}

A variação periódica do nível das águas éo principal fator que determina a comunidade de organismos aquáticos presente em rios com planícies alagadas. Muitos estudos na Amazônia são desenvolvidos nas várzeas próximas à cidade de Manaus, mas, comparações entre essas informaçôes são dificultadas pela ausência de padronização na denominação das diferentes etapas do ciclo de cheia-seca. Este trabalho teve como objetivo a identificação e a padronização da nomenclatura das diferentes fases do ciclo hidrológico para possibilitar análises que envolvam resultados de mais de um ano e de mais de um local. Os dados do nível da água do rio Negro, coletados no porto de Manaus foram utilizados para o desenvolvimento da metodologia. São propostos valores da cota do rio Negro para limitar os quatro períodos hidrológicos (enchente, cheia, vazante e seca) e definir a intensidade da cheia e da seca. O número de dias de cada um dos períodos hidrológicos foi obtido e foi estimada a duração para períodos considerados típicos, longos e curtos. Considerando que modificações abióticas e bióticas do meio estão relacionadas com as mudanças no nível da água, a identificação e a padronização da nomenclatura dos períodos hidrológicos mostraram-se extremamente úteis para uma primeira análise de informações biológicas dos organismos da várzea.

\section{PALAVRAS-CHAVE}

Ciclo hidrológico, várzea, rio Solimōes-Amazonas, Amazônia central.

\section{Proposal for rapid identification of the hydrological periods in lowland areas along the Solimoes Amazon river in the proximity of Manaus}

\begin{abstract}
Seasonal variations of the water level is the most important factor in determining the community of aquatic organisms present in flooded areas in the Amazon. Many scientific projects developed in this region were and still are being carried out in flooded areas near the city of Manaus; however, data can rarely be compared due to a non-standardized nomenclature used to describe the various phases of the flood pulse. The present paper aims to identify and standardize the terminology used to define a hydrological cycle with the purpose of allowing for the analysis of data from different places and multiple years. Serial data of the Negro River water height taken from the local harbor was considered for the estimations. Values of the water height are proposed in order to define the four hydrological periods (rising, flood, lowing, dry) and the intensity offlood and dry periods. A typical, long and short hydrological period was also defined in number of days based on the duration of each period. Considering that environmental abiotic and biotic modifications are related to water level fluctuation, the standardization of the hydrological nomenclature may become a useful tool for ecological data analysis.
\end{abstract}

KEY-WORDS

Hydrological cycle, floodplain, Solimões-Amazonas River, Central Amazon.

\footnotetext{
1 Instituto Nacional de Pesquisas da Amazônia, Coordenação de pesquisas em Biologia Aquática-CPBA. e-mail: mercedes@inpa.gov.br

2 Instituto Nacional de Pesquisas da Amazônia, Coordenação de pesquisas em Biologia Aquática-CPBA. e-mail: amadio@inpa.gov.br
} 
Em estudos de biologia e ecologia de organismos aquáticos da várzea, é amplamente aceita a hipótese de Junk et al. (1989), onde a variação periódica do nível das águas éo principal fator que determina a comunidade de organismos aquáticos presente em rios com planícies alagadas, causando modificações cíclicas, tanto abióticas quanto bióticas, nessas áreas inundáveis (Welcomme, 1985; Junk et al., 1989; Lowe-McConnell, 1999). Essa variação do nível da água é um acontecimento previsível para os organismos que vivem nas várzeas, que sincronizam os eventos de seu ciclo biológico para aproveitar os benefícios e/ou suportar as desvantagens decorrentes das mudanças no meio.

No rio Solimões-Amazonas, entretanto, ocorre uma variabilidade inter-anual na vazão, relacionada, em parte, às flutuaçôes no volume das chuvas. Diminuiçōes da pluviosidade na Amazônia estão parcialmente associadas ao fenômeno popularmente conhecido por "El Niño": o "El Niño" parece produzir seca ou vazante acentuada e "La Niña" ocasionar cheia intensa, (Welcomme, 1985; Richey et al., 1989; Nunes de Mello $\&$ Barros, 2001). Diferenças inter-anuais na composição de comunidades ícticas em ambientes de várzea têm sido relacionados à duração e intensidade dos períodos hidrológicos anteriores, como os trabalhos de Merona \& Gascuel (1993) e Cunico et al. (2002). A duração e intensidade de cada etapa dessa flutuação constituem fatores de imprevisibilidade para os organismos da várzea e que pode comprometer o sucesso do recrutamento das espécies da várzea.

Muitos estudos na Amazônia são desenvolvidos nas proximidades da cidade de Manaus. Mas, quando há a necessidade de comparar as informaçôes de diferentes estudos que relacionam aspectos bioecológicos e ciclo hidrológico, a primeira dificuldade que surge está relacionada à denominação não padronizada dada às diferentes etapas deste ciclo de cheiaseca.
Um ciclo hidrológico completo, em geral, é subdividido em quatro períodos, definidos segundo critérios hidrológicos: seca, enchente, cheia, e vazante. Mas, como determinar quando termina a seca e começa a enchente, por exemplo, quando a duração e intensidade do ciclo e de cada período hidrológico variam de ano para ano? Como classificar e padronizar os períodos quando informaçôes de mais de um local e ano são comparadas?

Este trabalho surgiu da necessidade de uma padronização das diferentes fases do ciclo hidrológico para que essa informação pudesse ser usada nos diversos segmentos desenvolvidos no estudo da biologia dos organismos da várzea da área do Catalão, onde não há serviço de medição do nível da água do rio. Essa padronização possibilita, ainda, o desenvolvimento de comparaçôes temporais e espaciais que envolvam dados coletados durante um intervalo de tempo superior a um ano e/ou proveniente de vários locais.

Os dados do nível da água do rio Negro, coletados no porto de Manaus, foram utilizados para o desenvolvimento da metodologia, assumindo as seguintes premissas: devido ao efeito do represamento causado pelo rio Solimōes/Amazonas, as variações do nível da água no rio Negro, medido no porto de Manaus e no rio Solimões/Amazonas são de magnitude semelhante nas áreas dentro de um perímetro aproximado de $200 \mathrm{~km}$ a partir de Manaus (Bayley, 1982; Guillaumet et al., 1988). Métodos de estatística descritiva foram utilizados para a caracterização dos períodos hidrológicos.

A cota de $23 \mathrm{~m}$ foi o valor médio encontrado para os 36.886 registros diários do nível do rio Negro obtidos no porto de Manaus entre 1903 e 2003; Annibal (1983) considerou esta mesma cota como valor médio de inundação Bankfull capaz de alagar as matas de várzea do Sistema Lago do Rei com cerca de um metro de profundidade.

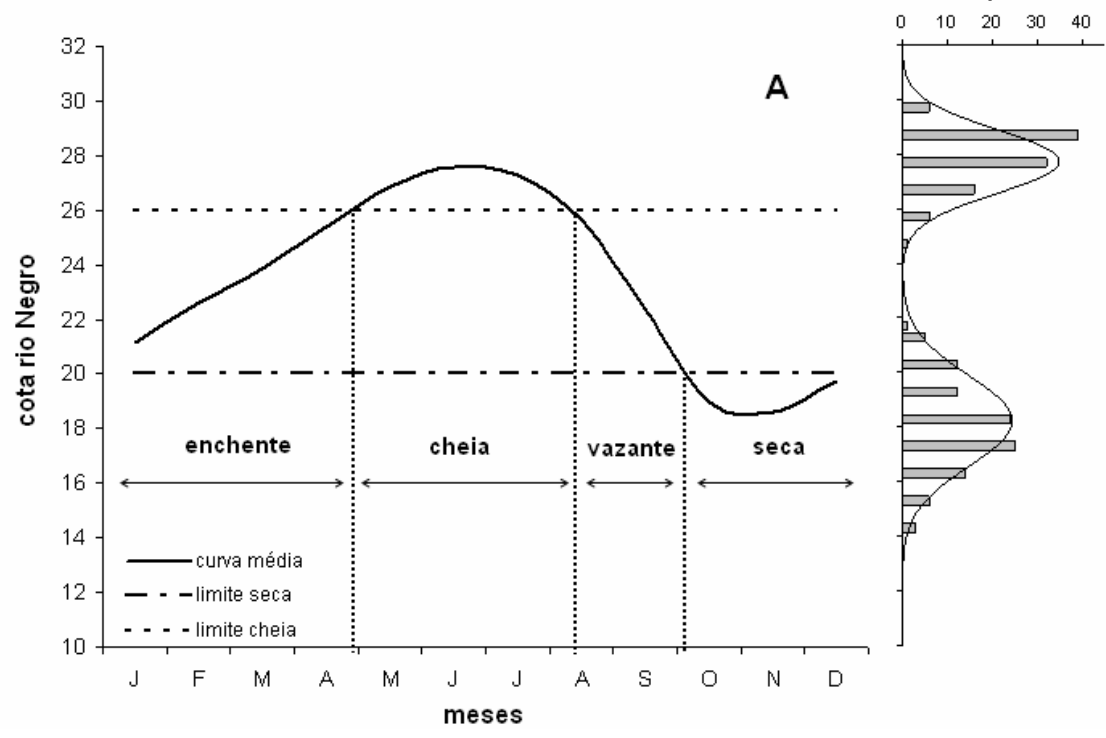

B

Figura 1 - Curva dos valores médios mensais do nível de água do rio Negro, indicando os quatro períodos hidrológicos definidos neste trabalho (A) e a frequência de ocorrência dos valores mínimos e máximos do nível da água obtidos para cada um dos 101 anos analisados (B). 
Annibal (com pess.) também utilizou para o lago do Rei, valores entre 21 e $25 \mathrm{~m}$ como limítrofes da seca e da cheia, respectivamente: dois metros abaixo e acima da cota 23 , mas não explica as bases para a adoção desses limites. Procurando limites para seca e cheia menos subjetivos que os adotados por esse autor, foram desenvolvidos os seguintes procedimentos apresentados abaixo.

Assumindo que a variabilidade na altura do fluxo pode ser estabelecida por uma distribuição de frequência (Welcomme,1985), foi identificada a menor e a maior cota registrada para cada um dos 101 anos. Foi calculada a freqüência, a média e o desvio padrão para as cotas mínimas e máximas, depois de verificada a normalidade da distribuição (Figura 1B).

Foi considerado para delimitar o período de cheia, o valor médio $(27,77 \mathrm{~m})$ menos o desvio padrão $(1,15)$ calculado para as cotas máximas anuais, que correspondeu aproximadamente à cota $26 \mathrm{~m}$. Igualmente, utilizando a média $(17,70 \mathrm{~m})$ acrescida do desvio padrão $(1,83)$ das cotas mínimas anuais, a cota de 20 $\mathrm{m}$ foi definida como valor limítrofe para o período de seca (Figura 1A).

Desta forma, para áreas do rio Solimões-Amazonas nas proximidades do município de Manaus, os períodos hidrológicos foram identificados, a partir dos valores obtidos no porto de Manaus, da seguinte forma:

Enchente: nível do rio ascendente, entre as cotas 20 e $26 \mathrm{~m}$

Cheia: cota igual ou superior a $26 \mathrm{~m}$

Vazante: nível do rio descendente, entre as cotas 26 e $20 \mathrm{~m}$ Seca: $\quad$ cota igual ou inferior a $20 \mathrm{~m}$

Como Ciclo Hidrológico, ficou definido o intervalo de tempo que compreende os quatro períodos contíguos acima citados (duração de $365 \pm 37$ dias = média \pm desvio padrão).

Nesse perímetro de $200 \mathrm{~km}$ a partir de Manaus, para o qual essa metodologia é proposta, a topografia de diferentes locais não é uniforme e ocorre uma variação temporal na entrada e recuo das águas do pulso de inundação nos diversos ambientes de várzea. Para minimizar parcialmente essas diferenças temporais e espaciais entre os diferentes locais, optou-se pelo uso dos valores médios mensais para definir o período hidrológico na qual nossa amostragem biológica foi realizada.

Além de favorecer o agrupamento e comparação dos dados, com essa classificação foi possível determinar a duração e intensidade dos diferentes períodos, fatores que influenciam a biologia dos organismos. A definição de cheia intensa ou seca fraca, período prolongado ou curto, por exemplo, tem sido até agora feita de forma subjetiva. Este estudo propõe uma classificação baseada também nos valores diários do nível da água do rio Negro coletados no porto de Manaus.

Com a definição de uma cota para o início e término de cada período hidrológico, foi possível calcular a duração de cada um
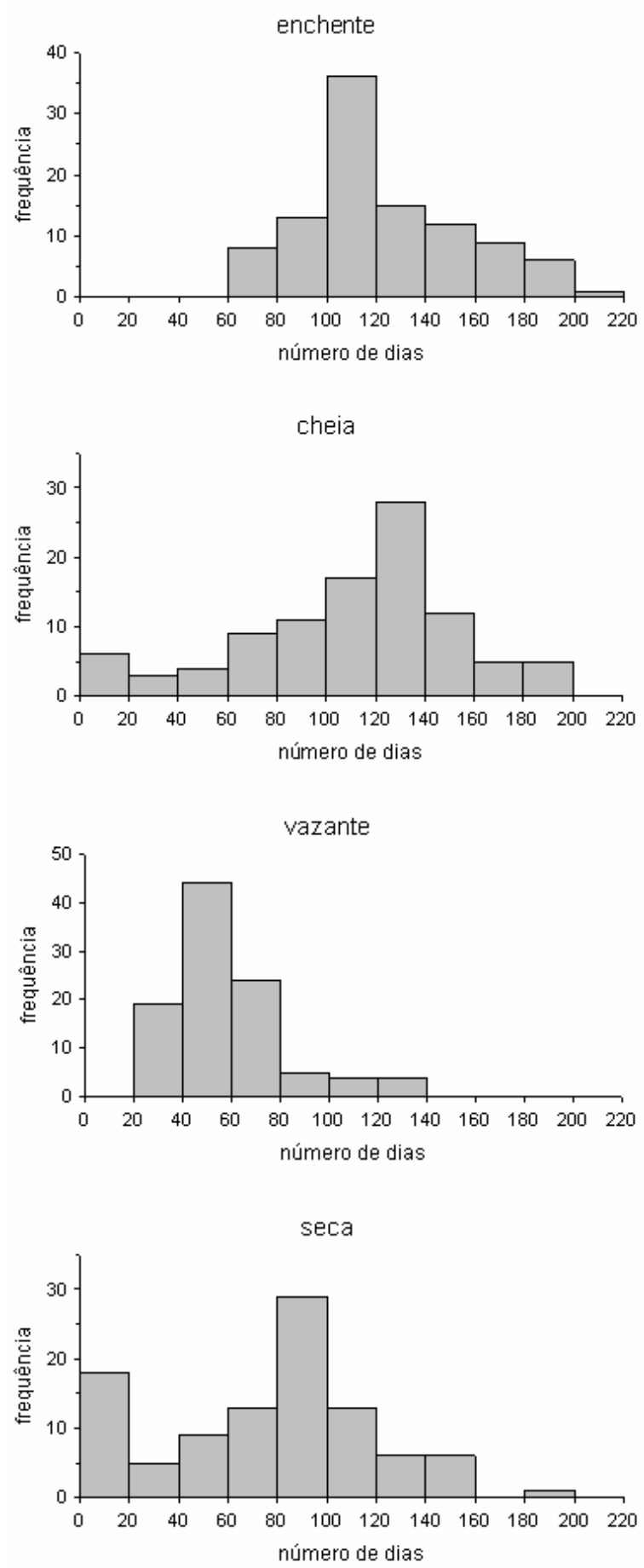

Figura 2 - Distribuição de freqüências da duração, em número de dias, dos períodos hidrológicos $(\mathrm{N}=100)$. 
dos períodos, em número de dias, para cada um dos 100 anos (1903 a 2003). O ano de 1926, por ter apresentado muito pouca variação no nível da água ao longo do tempo, não foi considerado nas análises.

Para descrever a variação na duração dos períodos hidrológicos, foi obtida uma distribuição de frequiência do número de dias, agrupados em classes de vinte dias, e calculada a média e o desvio padrão para cada período, após verificada a normalidade dessa distribuição (Figura 2). Com essas informaçōes, definimos a duração típica para cada um dos períodos; como os dados de vazante não apresentaram distribuição normal, a definição de duração padrão foi baseada no histograma de frequência deste período.

Em síntese, de acordo com os resultados deste trabalho, são propostas as seguintes definiçōes para os períodos hidrológicos no rio Solimões/Amazonas dentro de um perímetro de $200 \mathrm{~km}$, a partir de Manaus:
Quanto à duração ( $\mathrm{x}$ = número de dias):

Período típico longo curto

Enchente 90 a 160 dias Mais de 160 dias Menos de 90 dias Cheia 60 a 160 dias Mais de 160 dias Menos de 60 dias Vazante 30 a 70 dias Mais de 70 dias Menos de 30 dias Seca $\quad 30$ a 120 dias Mais de 120 dias Menos de 30 dias

Para a determinação da intensidade da seca e da cheia, foi utilizado o intervalo de confiança da média $(\mathrm{p}=0,05)$ dos valores das cotas mínimas e máximas obtidas para cada um dos 101 anos:

$\begin{array}{llll}\text { Período } & \text { típico } & \text { forte } & \text { fraco } \\ \text { Cheia } & \text { entre 27 e } & \text { Maior } & \text { Menor } \\ \text { (cota máxima) } & 28 \mathrm{~m} & \text { que } 28 \mathrm{~m} & \text { que } 27 \mathrm{~m} \\ \text { Seca } & \text { entre } 17 \mathrm{e} & \text { Menor } & \text { Maior } \\ \text { (cota mínima) } & 19 \mathrm{~m} & \text { que } 17 \mathrm{~m} & \text { que } 19 \mathrm{~m}\end{array}$

Tabela 1 - Valores e classificação da intensidade e duração dos períodos hidrológicos nas áreas de várzea próximas a Manaus, para os anos de 1953 a 2003. (classif = classificação; $T$ = típico; $F=$ forte; $F r=$ fraco; $L=$ longo; $C=$ curto).

\begin{tabular}{|c|c|c|c|c|c|c|c|c|c|c|c|c|c|}
\hline \multirow{3}{*}{ ANO } & \multicolumn{4}{|c|}{ INTENSIDADE } & \multicolumn{8}{|c|}{ DURAÇÃO } & \multirow{3}{*}{$\begin{array}{c}\text { ciclo hidrológico } \\
n^{\circ} \text { dias }\end{array}$} \\
\hline & \multicolumn{2}{|c|}{ seca } & \multicolumn{2}{|c|}{ cheia } & \multicolumn{2}{|c|}{ enchente } & \multicolumn{2}{|c|}{ cheia } & \multicolumn{2}{|c|}{ vazante } & \multicolumn{2}{|c|}{ seca } & \\
\hline & cota & classif & cota & classif & $\mathrm{n}^{\circ}$ dias & classif & $\mathrm{n}^{\circ}$ dias & classif & $\mathrm{n}^{\circ}$ dias & classif & $\mathrm{n}^{\circ}$ dias & classif & \\
\hline 1953 & 17.06 & $T$ & 29.69 & $\mathrm{~F}$ & 76 & C & 184 & $\mathrm{~L}$ & 34 & $\mathrm{~T}$ & 56 & $\mathrm{~T}$ & 350 \\
\hline 1954 & 17.63 & $\mathrm{~T}$ & 28.49 & $\mathrm{~F}$ & 119 & $\mathrm{~T}$ & 139 & $\mathrm{~T}$ & 42 & $\mathrm{~T}$ & 96 & $\mathrm{~T}$ & 396 \\
\hline 1955 & 16.03 & $\mathrm{~F}$ & 28.53 & $\mathrm{~F}$ & 97 & $\mathrm{~T}$ & 135 & $\mathrm{~T}$ & 32 & $\mathrm{~T}$ & 130 & $\mathrm{~L}$ & 394 \\
\hline 1956 & 20.90 & $\mathrm{Fr}$ & 27.65 & $\mathrm{~T}$ & 81 & C & 120 & $T$ & 67 & $\mathrm{~T}$ & 0 & C & 268 \\
\hline 1957 & 16.51 & $\mathrm{~F}$ & 27.33 & $T$ & 200 & $\mathrm{~L}$ & 90 & $\mathrm{~T}$ & 35 & $\mathrm{~T}$ & 85 & $T$ & 410 \\
\hline 1958 & 14.74 & $\mathrm{~F}$ & 27.58 & $\mathrm{~T}$ & 133 & $\mathrm{~T}$ & 100 & $\mathrm{~T}$ & 48 & $\mathrm{~T}$ & 85 & $\mathrm{~T}$ & 366 \\
\hline 1959 & 18.66 & $\mathrm{~T}$ & 27.71 & $\mathrm{~T}$ & 139 & $\mathrm{~T}$ & 112 & $\mathrm{~T}$ & 49 & $\mathrm{~T}$ & 45 & $\mathrm{~T}$ & 345 \\
\hline 1960 & 18.33 & $\mathrm{~T}$ & 27.56 & $\mathrm{~T}$ & 164 & $\mathrm{~L}$ & 92 & $\mathrm{~T}$ & 58 & $\mathrm{~T}$ & 67 & $\mathrm{~T}$ & 381 \\
\hline 1962 & 17.15 & $T$ & 28.33 & $\mathrm{~F}$ & 114 & $T$ & 136 & $T$ & 34 & $T$ & 135 & $\mathrm{~L}$ & 419 \\
\hline 1963 & 13.64 & $\mathrm{~F}$ & 27.31 & $\mathrm{~T}$ & 77 & C & 84 & $\mathrm{~T}$ & 45 & $\mathrm{~T}$ & 192 & $\mathrm{~L}$ & 398 \\
\hline 1964 & 18.41 & $\mathrm{~T}$ & 25.91 & $\mathrm{Fr}$ & 116 & $\mathrm{~T}$ & 0 & C & 100 & $\mathrm{~L}$ & 100 & $\mathrm{~T}$ & 316 \\
\hline 1965 & 16.00 & $\mathrm{~F}$ & 26.58 & $\mathrm{Fr}$ & 109 & $\mathrm{~T}$ & 60 & $\mathrm{~T}$ & 65 & $\mathrm{~T}$ & 95 & $\mathrm{~T}$ & 329 \\
\hline 1966 & 16.76 & $\mathrm{~F}$ & 26.41 & $\mathrm{Fr}$ & 160 & $\mathrm{~T}$ & 35 & $C$ & 71 & $L$ & 115 & $\mathrm{~T}$ & 381 \\
\hline 1967 & 16.18 & $\mathrm{~F}$ & 27.91 & $\mathrm{~T}$ & 90 & $\mathrm{~T}$ & 132 & $\mathrm{~T}$ & 44 & $\mathrm{~T}$ & 111 & $\mathrm{~T}$ & 377 \\
\hline 1968 & 21.40 & $\mathrm{Fr}$ & 27.13 & $\mathrm{~T}$ & 103 & $\mathrm{~T}$ & 72 & $\mathrm{~T}$ & 124 & $\mathrm{~L}$ & 0 & C & 299 \\
\hline 1969 & 16.86 & $\mathrm{~F}$ & 27.40 & $\mathrm{~T}$ & 157 & $\mathrm{~T}$ & 95 & $\mathrm{~T}$ & 60 & $\mathrm{~T}$ & 111 & $\mathrm{~T}$ & 423 \\
\hline 1970 & 18.19 & $T$ & 28.31 & $\mathrm{~F}$ & 89 & C & 135 & $T$ & 62 & $T$ & 80 & $\mathrm{~T}$ & 366 \\
\hline 1971 & 21.14 & $\mathrm{Fr}$ & 29.11 & $\mathrm{~F}$ & 72 & C & 169 & $\mathrm{~L}$ & 57 & $\mathrm{~T}$ & 0 & $C$ & 298 \\
\hline 1972 & 20.02 & $\mathrm{Fr}$ & 28.70 & $\mathrm{~F}$ & 132 & $T$ & 164 & $\mathrm{~L}$ & 74 & $\mathrm{~L}$ & 0 & C & 370 \\
\hline 1974 & 21.84 & $\mathrm{Fr}$ & 28.46 & $\mathrm{~F}$ & 133 & $\mathrm{~T}$ & 162 & $\mathrm{~L}$ & 89 & $\mathrm{~L}$ & 0 & C & 384 \\
\hline
\end{tabular}


Tabela 1 - continuação

\begin{tabular}{|c|c|c|c|c|c|c|c|c|c|c|c|c|c|}
\hline \multirow{3}{*}{ ANO } & \multicolumn{4}{|c|}{ INTENSIDADE } & \multicolumn{8}{|c|}{ DURAÇÃO } & \multirow{3}{*}{$\begin{array}{c}\text { ciclo hidrológico } \\
n^{\circ} \text { dias }\end{array}$} \\
\hline & \multicolumn{2}{|c|}{ seca } & \multicolumn{2}{|c|}{ cheia } & \multicolumn{2}{|c|}{ enchente } & \multicolumn{2}{|c|}{ cheia } & \multicolumn{2}{|c|}{ vazante } & \multicolumn{2}{|c|}{ seca } & \\
\hline & cota & classif & cota & classif & $n^{\circ}$ dias & classif & $n^{\circ}$ dias & classif & $n^{\circ}$ dias & classif & $\mathrm{n}^{\circ}$ dias & classif & \\
\hline 1975 & 19.32 & $\mathrm{Fr}$ & 29.11 & $\mathrm{~F}$ & 105 & T & 182 & $\mathrm{~L}$ & 51 & $\mathrm{~T}$ & 29 & C & 367 \\
\hline 1976 & 18.05 & $\mathrm{~T}$ & 29.61 & $\mathrm{~F}$ & 105 & T & 159 & T & 42 & T & 76 & $T$ & 382 \\
\hline 1977 & 20.66 & $\mathrm{Fr}$ & 28.45 & $\mathrm{~F}$ & 113 & T & 127 & T & 51 & T & 0 & C & 291 \\
\hline 1978 & 20.12 & $\mathrm{Fr}$ & 28.11 & $\mathrm{~F}$ & 187 & L & 121 & T & 110 & L & 0 & C & 418 \\
\hline 1979 & 17.44 & $\mathrm{~T}$ & 28.23 & $\mathrm{~F}$ & 135 & T & 108 & T & 47 & T & 97 & T & 387 \\
\hline 1980 & 17.68 & $\mathrm{~T}$ & 26.00 & $\mathrm{Fr}$ & 184 & L & 3 & C & 69 & T & 71 & $\mathrm{~T}$ & 327 \\
\hline 1981 & 17.24 & $\mathrm{~T}$ & 26.86 & $\mathrm{Fr}$ & 167 & L & 90 & T & 51 & T & 98 & T & 406 \\
\hline 1982 & 18.28 & T & 28.97 & $\mathrm{~F}$ & 100 & T & 134 & T & 48 & T & 59 & T & 341 \\
\hline 1983 & 17.08 & $\mathrm{~T}$ & 26.52 & $\mathrm{Fr}$ & 159 & T & 46 & C & 58 & T & 116 & T & 379 \\
\hline 1984 & 19.58 & $\mathrm{Fr}$ & 28.03 & $\mathrm{~F}$ & 115 & T & 128 & T & 60 & T & 54 & T & 357 \\
\hline 1985 & 19.74 & $\mathrm{Fr}$ & 26.27 & $\mathrm{Fr}$ & 189 & L & 28 & C & 126 & L & 12 & C & 355 \\
\hline 1986 & 21.40 & $\mathrm{Fr}$ & 28.14 & $\mathrm{~F}$ & 140 & T & 130 & T & 47 & T & 0 & C & 317 \\
\hline 1987 & 17.99 & $\mathrm{~T}$ & 27.91 & T & 177 & L & 112 & T & 57 & T & 85 & T & 431 \\
\hline 1988 & 17.82 & $\mathrm{~T}$ & 27.78 & T & 137 & T & 104 & T & 37 & T & 69 & T & 347 \\
\hline 1989 & 19.55 & $\mathrm{Fr}$ & 29.99 & $F$ & 103 & $\mathrm{~T}$ & 182 & L & 106 & L & 34 & $\mathrm{~T}$ & 425 \\
\hline 1990 & 16.32 & $\mathrm{~F}$ & 28.23 & $\mathrm{~F}$ & 68 & C & 138 & $\mathrm{~T}$ & 42 & $\mathrm{~T}$ & 67 & T & 315 \\
\hline 1991 & 16.07 & $F$ & 28.06 & $\mathrm{~F}$ & 138 & $\mathrm{~T}$ & 122 & $\mathrm{~T}$ & 37 & $\mathrm{~T}$ & 155 & L & 452 \\
\hline 1992 & 17.56 & $\mathrm{~T}$ & 25.42 & $\mathrm{Fr}$ & 78 & C & 0 & C & 121 & L & 84 & $\mathrm{~T}$ & 283 \\
\hline 1993 & 19.47 & $\mathrm{Fr}$ & 28.76 & $F$ & 99 & $\mathrm{~T}$ & 148 & $\mathrm{~T}$ & 60 & $\mathrm{~T}$ & 25 & C & 332 \\
\hline 1994 & 19.06 & $\mathrm{Fr}$ & 29.05 & $F$ & 118 & $T$ & 182 & L & 62 & $\mathrm{~T}$ & 32 & $\mathrm{~T}$ & 394 \\
\hline 1995 & 15.06 & $\mathrm{~F}$ & 27.16 & $\mathrm{~T}$ & 158 & T & 78 & $\mathrm{~T}$ & 36 & $T$ & 97 & $T$ & 369 \\
\hline 1996 & 19.14 & $\mathrm{Fr}$ & 28.54 & $\mathrm{~F}$ & 102 & $\mathrm{~T}$ & 150 & $\mathrm{~T}$ & 42 & $\mathrm{~T}$ & 56 & $\mathrm{~T}$ & 350 \\
\hline 1997 & 14.34 & $F$ & 28.84 & $F$ & 116 & $T$ & 139 & $T$ & 35 & $T$ & 122 & L & 412 \\
\hline 1998 & 15.03 & $\mathrm{~F}$ & 27.58 & $\mathrm{~T}$ & 116 & $\mathrm{~T}$ & 93 & $\mathrm{~T}$ & 37 & $\mathrm{~T}$ & 111 & $\mathrm{~T}$ & 357 \\
\hline 1999 & 16.95 & $F$ & 29.30 & $F$ & 62 & C & 183 & L & 32 & $T$ & 88 & $T$ & 365 \\
\hline 2000 & 18.57 & $T$ & 28.62 & $F$ & 106 & $T$ & 136 & $T$ & 64 & $T$ & 54 & $T$ & 360 \\
\hline 2001 & 16.81 & $\mathrm{~F}$ & 28.21 & $\mathrm{~F}$ & 94 & $\mathrm{~T}$ & 134 & $\mathrm{~T}$ & 50 & $\mathrm{~T}$ & 82 & $\mathrm{~T}$ & 360 \\
\hline 2002 & 17.19 & $\mathrm{~T}$ & 28.91 & $\mathrm{~F}$ & 108 & $\mathrm{~T}$ & 144 & $\mathrm{~T}$ & 39 & $\mathrm{~T}$ & 47 & $\mathrm{~T}$ & 338 \\
\hline 2003 & 19.01 & $\mathrm{Fr}$ & 28.27 & $\mathrm{~F}$ & 153 & T & 121 & $\mathrm{~T}$ & 45 & $\mathrm{~T}$ & 86 & $\mathrm{~T}$ & 405 \\
\hline
\end{tabular}

Informações baseadas nos valores diários do nível da água do rio Negro no porto de Manaus e nos dados obtidos pela metodologia descrita no trabalho

Como a enchente e a vazante são definidas dentro de um intervalo de cota (20 e $26 \mathrm{~m})$, a intensidade não pode ser calculada para esses dois períodos.

Tendo como base os dados diários do nível da água do porto de Manaus, disponibilizados pelo Serviço de Engenharia da Sociedade de Navegação, Porto e Hidrovias do Amazonas, e a metodologia acima descrita, apresentamos, na Tabela 1, a classificação da duração e intensidade dos períodos hidrológicos dos anos de 1953 a 2003.
A identificação e, principalmente, a padronização da nomenclatura têm se mostrado extremamente úteis para uma primeira análise de informaçóes biológicas dos organismos da várzea. $\mathrm{O}$ método possibilita o agrupamento e comparação dos dados biológicos, mesmo quando medidas do nível da água são inexistentes, mas não substitui uma caracterização hidrológica, como qualidade da água, para uma descrição da dinâmica local do pulso de inundação. 


\section{BIBLIOGRAFIA CITADA}

Annibal, S.R.P. 1983. Avaliação Bio-Ecológica e Pesqueira das "Pescadas" (Plagioscion squamosissimus Heckel, 1940) e Plagioscion montei, do "Sistema Lago do Rei - Ilha do Careiro-AM-Brasil. Dissertação de Mestrado, Instituto Nacional de Pesquisas da Amazônia/ Universidade Federal do Amazonas. Manaus, Amazonas. 134pp.

Bayley, P.B. 1982. Central Amazon Fish Populations: Biomass, Productions and some Dynamics Characteristics. Tese de Doutorado. Dalhousie University, Halifax. 330pp.

Cunico, A.M.; Graça, W.J.; Veríssimo. S.; Bini, L.M. 2002. Influência do nível hidrológico sobre a assembléia de peixes em lagoa sazonalmente isolada da planície de inundação do alto rio Paraná. Acta Scientiarum, 24 (2): 383-389.

Guillaumet, J.-L., Oliveira Marques. Fo. A., Tancrede, A.C., Cunha, H.B., Senna, R.C., Igreja, S.M.S.; Merona, B. de (1988) Eléments d'hydrologie. In: Conditions Écologiques et Économiques de la Production d'un Île De Várzea: l'Île de Careiro. Rapport Terminal. ORSTOM/INPA/CEE: 21-35.

Junk, W.J.; Bayley, P.B.; Sparks, R.E. 1989. The flood concept in river-floodplain systems. In: Dodge, D. P. (Ed.) - Proceedings of the International Large River Symposium. Lan. Spec. Publ. Fish. Aquat. Sci.: 110-127.
Lowe-McConnell, R.H. 1999. Estudos Ecológicos de Comunidades de Peixes Tropicais. EDUSP. São Paulo. 536pp.

Merona, B.; Gascuel, D. 1993. The effects of flood regime and fishing effort on the overall abundance of an exploited fish community in the Amazon floodplain. Aquat..Living Resour., 6: 97-108.

Nunes de Mello, J.A.; Barros, W.G. 2001. Enchentes e vazantes do rio Negro medidas no porto de Manaus, Amazonas, Brasil. Acta Amazonica, 31(2): 331-337

Richey, J.E.; Nobre, C.; Deser, C. (1989) - Amazon River discharge and climate variability: 1903 to 1985 . Science, 246: 101-103.

Welcomme, R.L. 1985. River fisheries. FAO Fish. Tech. Pap., (262): 330.

Recebido em 01/03/2006

Aceito em 28/02/2007 